\title{
How can additional ultrasonography screening improve the detection of occult breast cancer in women with dense breasts?
}

\author{
Parisa Pishdad ${ }^{1,2 A, C, D}$, Ameneh Moosavi ${ }^{2 B}$, Reza Jalli1,20, Fariba Zarei ${ }^{1,2 A, F}$, Mahdi Saeedi-Moghadam ${ }^{1 B, F}$, \\ Banafsheh Zeinali-Rafsanjani ${ }^{1,3 B, D, E}$ \\ 'Medical Imaging Research Center, Shiraz University of Medical Sciences, Shiraz, Iran \\ 2Department of Radiology, Shiraz University of Medical Sciences, Shiraz, Iran \\ ${ }^{3}$ Nuclear Medicine and Molecular Imaging Research Center, Shiraz University of Medical Sciences, Shiraz, Iran
}

\section{Abstract}

\begin{abstract}
Purpose: Although mammography is a gold standard for breast cancer screening, the number of cancers that cannot be detected with mammography is substantial, especially in dense-breast (DB) women. Breast sonography can be a useful and powerful screening tool in these cases. The aim of this study is to assess the application of whole-breast sonography in the evaluation of breast lesions in women with DB tissue and estimate its accuracy in comparison with mammography.
\end{abstract}

Material and methods: A total of 207 asymptomatic DB women participated in this study. The breast tissue density was assessed using ACR BI-RADS. Patients underwent high-resolution ultrasonography of the breast in addition to physical examination and mammography. Different risk factors were also assessed.

Results: 152 of 207 (73.4\%) cases who had mammography performed had DB, and 55 (26.6\%) cases had very dense breasts (very DBs). None of the cases had a positive history of malignancy, while $19 \%$ of them had a positive history of breast cancer in first- or second-degree relatives.

Conclusions: All findings were higher in cases with DB compared to very DBs except for fibroadenoma, which was detected more in cases with very DBs. Our study showed that the prevalence of different breast lesions had a significant relationship with the density of the breast. In our study, $48.3 \%$ of the cases were diagnosed with a lesion in their sonography result, although $81.0 \%$ of them were benign lesions, and the other $19.0 \%$ needed follow-up or biopsy evaluation. A substantial number of mammographically occult breast lesions, either benign or malignant, could be detected by ultrasound in DB tissue.

Key words: ultrasonography screening, occult breast cancer, risk factors.

\section{Introduction}

Breast cancer is the second most common cancer in women (after skin cancer). It is also considered as the most prevalent cause of mortality in women worldwide [1]. Breast cancer treatment is very costly, comprising various methods of chemotherapy, radiotherapy, or brachytherapy [2]. Screening is the first strategy for reducing breast cancer mortality and treatment cost [3].
Mammography is the standard screening method that has reduced breast cancer mortality in the last decades. However, the number of cancers that cannot be detected with mammography is substantial, especially in densebreasted women $[4,5]$. Mammography sensitivity in evaluating breast mass in the dense breast is as low as $30-48 \%$. Studies also show that the chance of breast cancer between screenings was almost 18 times higher in very dense breasts compared with fatty breasts [6].

\section{Correspondence address:}

Dr. Banafsheh Zeinali-Rafsanjani, Medical Imaging Research Center, Shiraz University of Medical Sciences, Shiraz, Iran, e-mail: b.zeinali.r@gmail.com Authors' contribution:

A Study design · B Data collection · C Statistical analysis · D Data interpretation · E Manuscript preparation · F Literature search · G Funds collection 
In the last year the American Cancer Society (ACS) suggested magnetic resonance imaging (MRI) as a screening tool for very high-risk cases. However, MRI is expensive and might cause risks due to the need for contrast media. Moreover, MRI has a lower specificity for breast cancer screening in comparison to mammography, so it has a higher rate of false positives, and consequently it needs further MRI follow-up [7].

There is a direct correlation between increased breast density and higher interval cancer rates, and there is a worse prognosis for these clinically detected cancers. Subsequently, it can deduce that dense breast tissue itself is a risk factor for breast cancer. Thus, techniques as a complement to mammography are required, principally for women with dense breasts [8].

Breast sonography can be a useful and powerful screening tool because it is widely accessible, easy to perform, free of detrimental side effects, and it does not require radiation or contrast agents. Studies have also revealed that performing breast sonography for patients with breast pain can decrease pain and anxiety [9].

Also, studies have shown that application of supplementary screening tools such as breast sonography increases the chance of detection of early node-negative invasive breast cancer in women with dense breast tissue.

The incidence rates of breast cancer in Asia are lower than in Europe and the United States; however, breast density is noticeably higher in Asian than in African American and white women. It implies that a different diagnostic routine of screening breast sonography should be considered in Asian women [10].

The purpose of this study is to evaluate the application of whole-breast sonography in the assessment of breast lesions in women with dense breast tissue, and to estimate its accuracy in comparison with mammography. We also investigated different factors that might influence the prevalence of different breast lesions.

\section{Material and methods}

During December 2015 and March 2016, 207 asymptomatic women participated in this study. The breast tissue densities of participants were designated as categories 3 or 4, using the Breast Imaging Reporting and Data System (BI-RADS). High-resolution ultrasonography of the breast was performed for all patients after mammography and physical examination.

All breast sonographies were performed by an experienced radiologist. The same radiologist who reviewed the screening mammography also performed screening sonography examinations.

All the mammograms were reviewed before screening sonography. The breast density was determined as stated by the gradation of the American College of Radiology (ACR) BI-RADS protocol on a scale from A to D. If the findings on mammography were normal and the breast density was defined as grades C-D, the patients were considered as appropriate cases for screening sonography.

The procedure was explained to all the patients in detail by the examining breast radiologist and when the patients received the results of the screening mammography. The patients provided verbal consent.

Prior to sonography, a physical examination was performed with appropriate anatomical positioning for all patients by the radiologist. The existence of a palpable abnormality led to the exclusion of the patient from the study.

All sonographies were performed with ATL 3000 or 5000 units by using 5-12 MHz bandwidth electronically focused transducers. Both breasts were thoroughly examined with overlapping radial and anti-radial, vertical and horizontal scans. To ensure the complete scan of all breast tissue, the retro-areolar region was scanned with angled views. depending on the size and texture of the breasts, the procedure time was varied between 4 and $15 \mathrm{~min}$ (mean: $7 \mathrm{~min}$ ). The unit was not equipped with elastography.

The sonographic findings were categorised as BIRADS I (no focal lesions), BIRADS II (fibrocystic change and simple cyst), BIRADS III (probably benign including complicated cyst, ductal ectasia, fibroadenoma), and BIRADS IV and $\mathrm{V}$ (suspicious or probably malignant lesions). In cases with more than one lesion, the final assessment was categorised as the highest BIRADS.

A questionnaire was used to gather information about the risk factors related to the prevalence of breast cancer. The risk factors included the duration of oral contraceptive pill (OCP) usage, the frequency of pregnancy, frequency of breastfeeding, previous history of cancer, and history of breast cancer in first- and second-degree relatives.

The $\chi^{2}$ or $t$-test was performed to analyse the results statistically. Appropriate Pearson correlation was carried out to determine the relationships between the variables. The $p$-value of less than 0.05 considered as statistically significant.

\section{Results}

A total of 207 women aged 32-62 years, mean \pm SD 43.7 \pm 6.5 years, participated in this study. Out of a total 207 performed mammographies, 152 cases $(73.4 \%)$ had dense and 55 cases (26.6\%) had very dense breast. None of the cases had a positive history of malignancy, while $19 \%$ of them had a positive record of breast cancer in first- or second-degree relatives. Table 1 shows the prevalence of the sonography findings. According to Table 1, 115 cases were normal, and there were 100 findings in 92 cases.

The benign lesions included fibrocystic change, simple cyst, and cluster microcyst (Figures 1 and 2); the prevalence of these lesions separately were 38,37 , and 6 , respectively. The majority of our subjects had normal and benign breast 
Table 1. The prevalence of different sonography findings

\begin{tabular}{|l|c|c|c|c|c|c|}
\hline Sonography result & Normal & Benign lesion* & Complicated cyst & Ductal ectasia & Fibroadenoma & Cancer \\
\hline Number & 115.0 & 81.0 & 4.0 & 6.0 & 8.0 & 1.0 \\
\hline Percentage & 55.5 & 39.1 & 2.9 & 1.9 & 3.9 & 0.5 \\
\hline
\end{tabular}

*Benign lesion includes fibrocystic change, simple cyst, and cluster microcyst
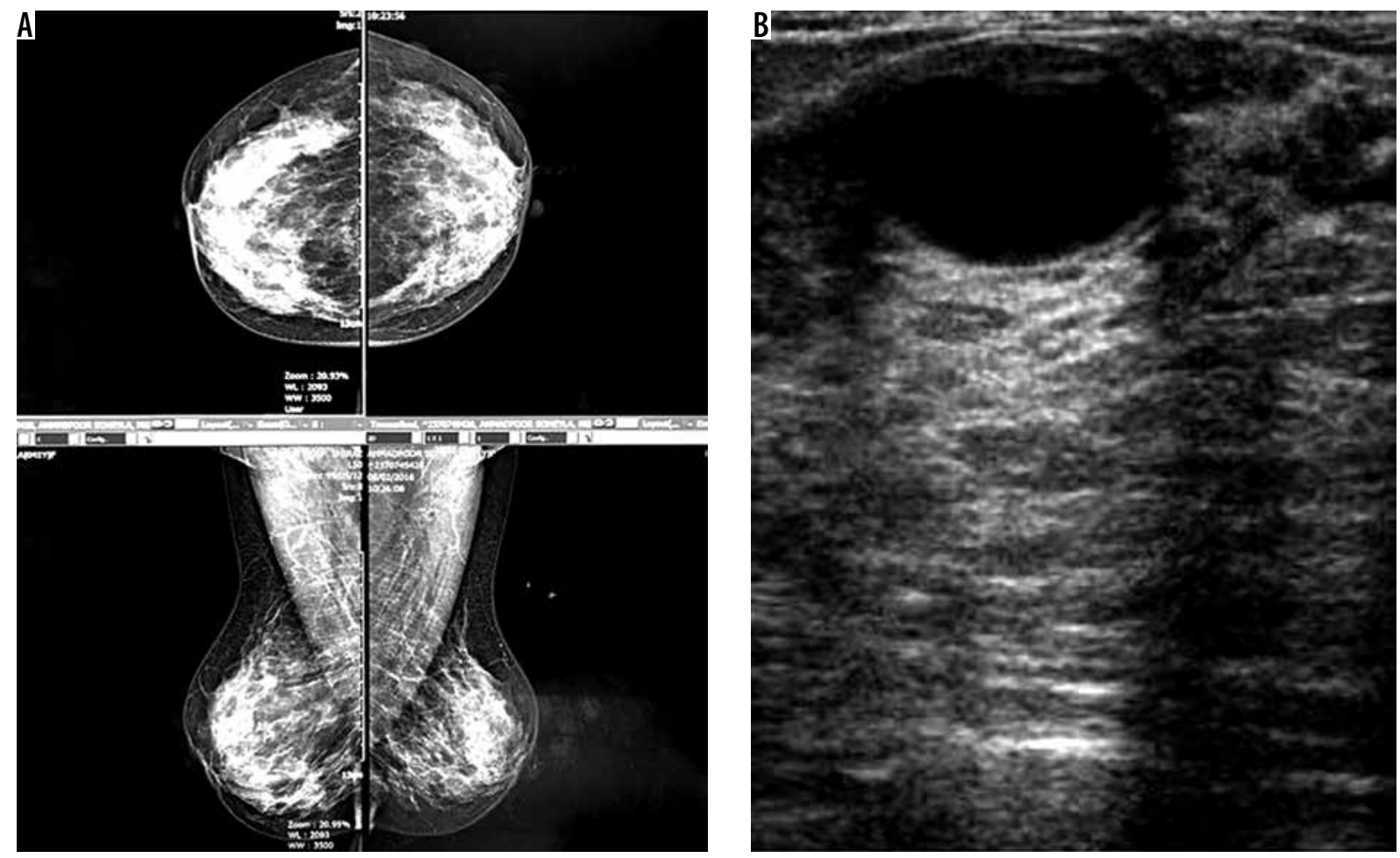

Figure 1. A) An asymptomatic woman with mammographically dense breast. B) Simple cyst in ultrasound image of the same patient
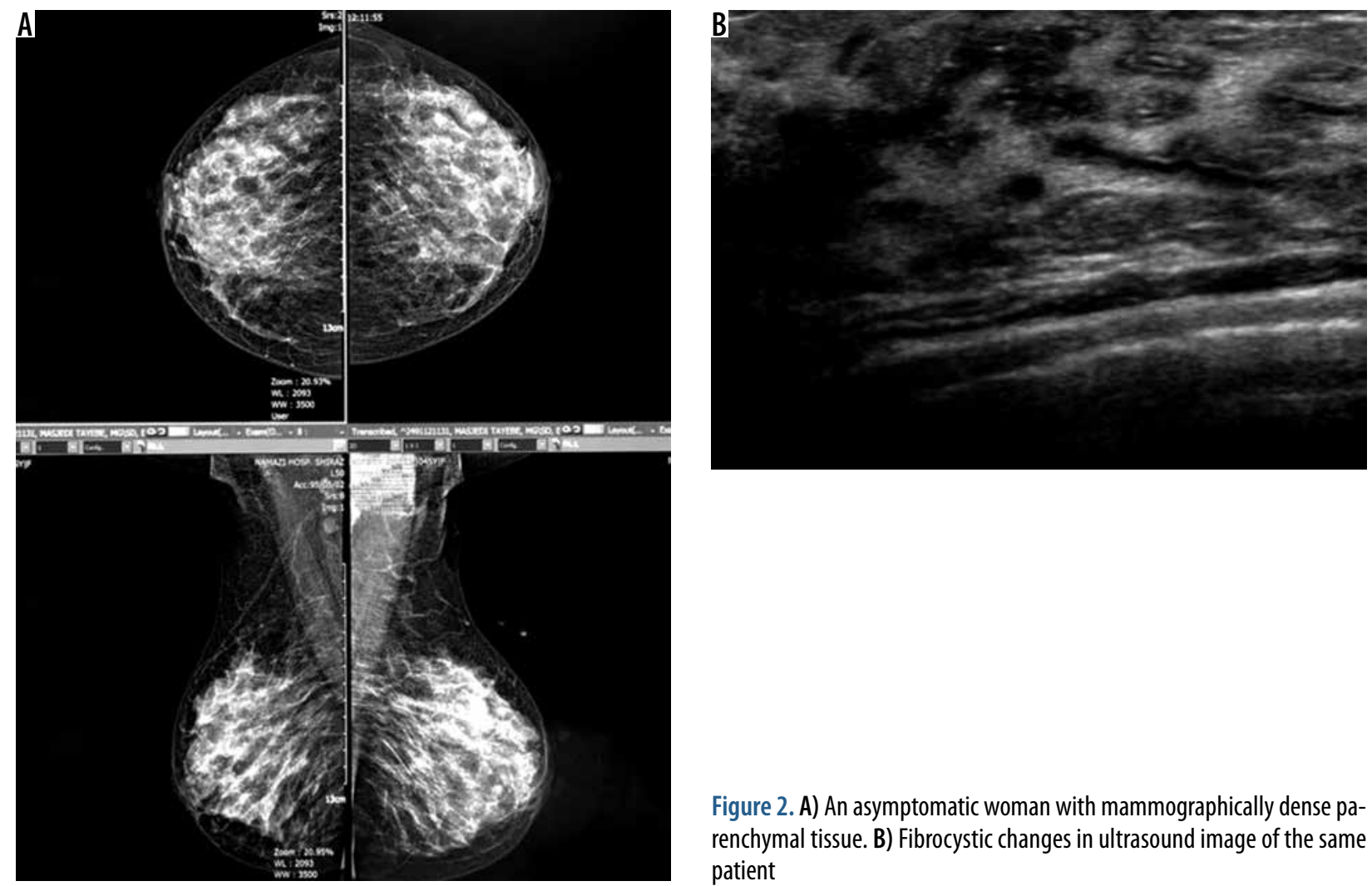

Figure 2. A) An asymptomatic woman with mammographically dense parenchymal tissue. B) Fibrocystic changes in ultrasound image of the same patient 


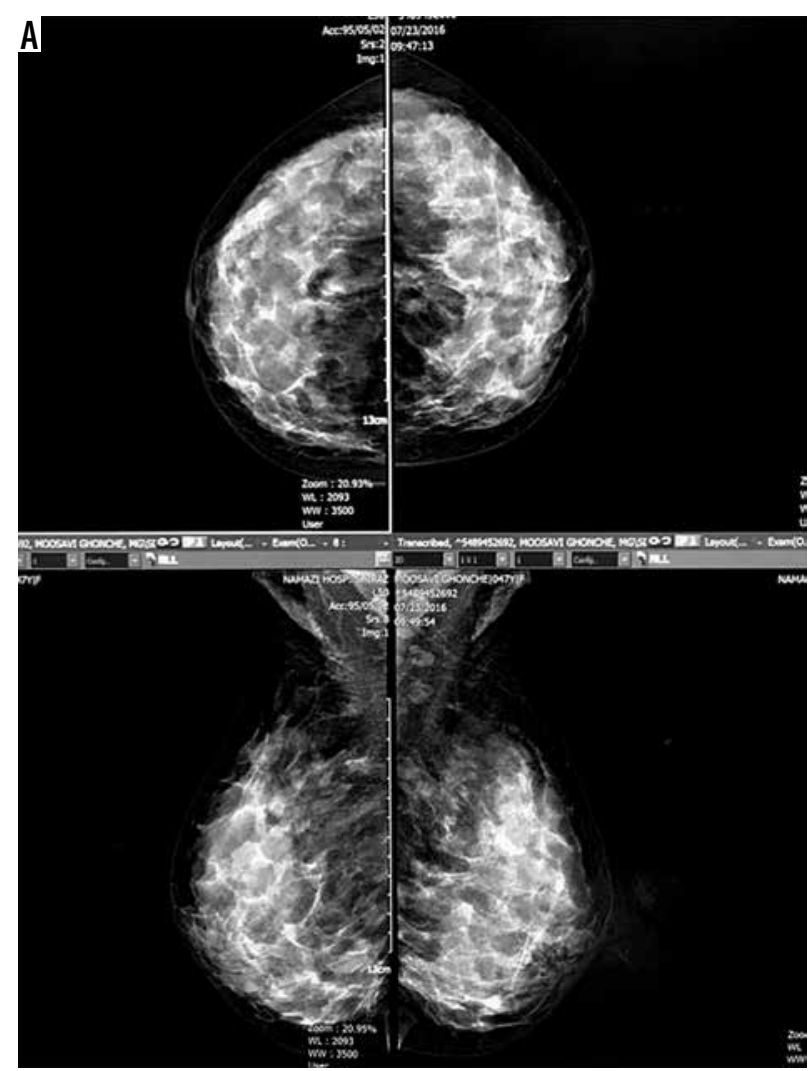

lesions. All cases with ductal ectasia had bilateral lesions (Figure 3). Nine cases underwent short-term follow-up at $6,12,24$, and 36 months without any biopsy and just by mammography and sonography.
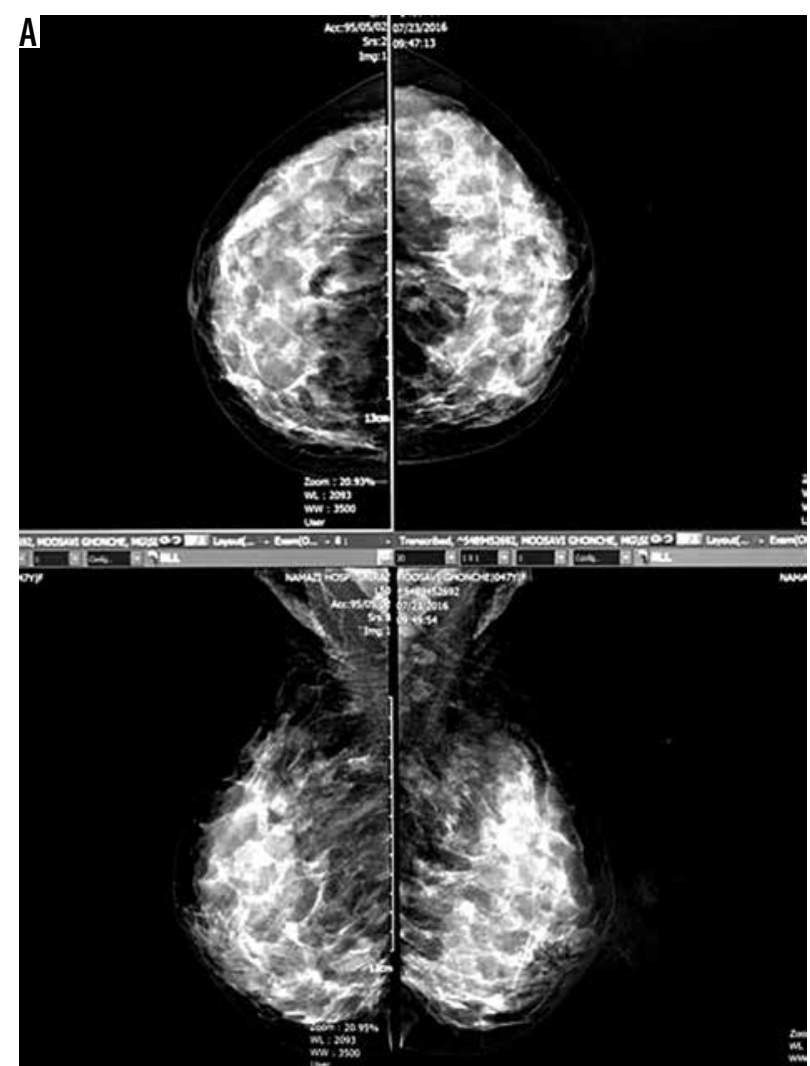

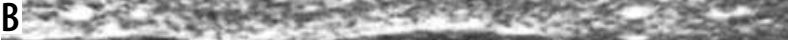

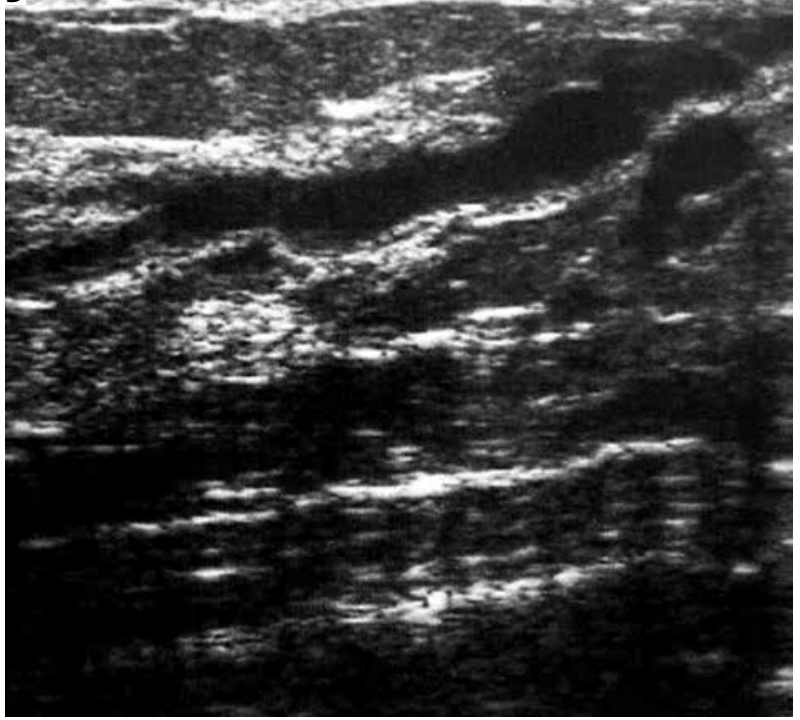

Figure 3. A) An asymptomatic mammographically dense breast. B) Ductal ectasia in ultrasound image of the same patient

Four cases had complicated cyst with no changes in size and no other new lesion during the follow-up time (Figure 4). Five cases were typically fibroadenoma (i.e. well-circumscribed, homogenous hypo echo, round to

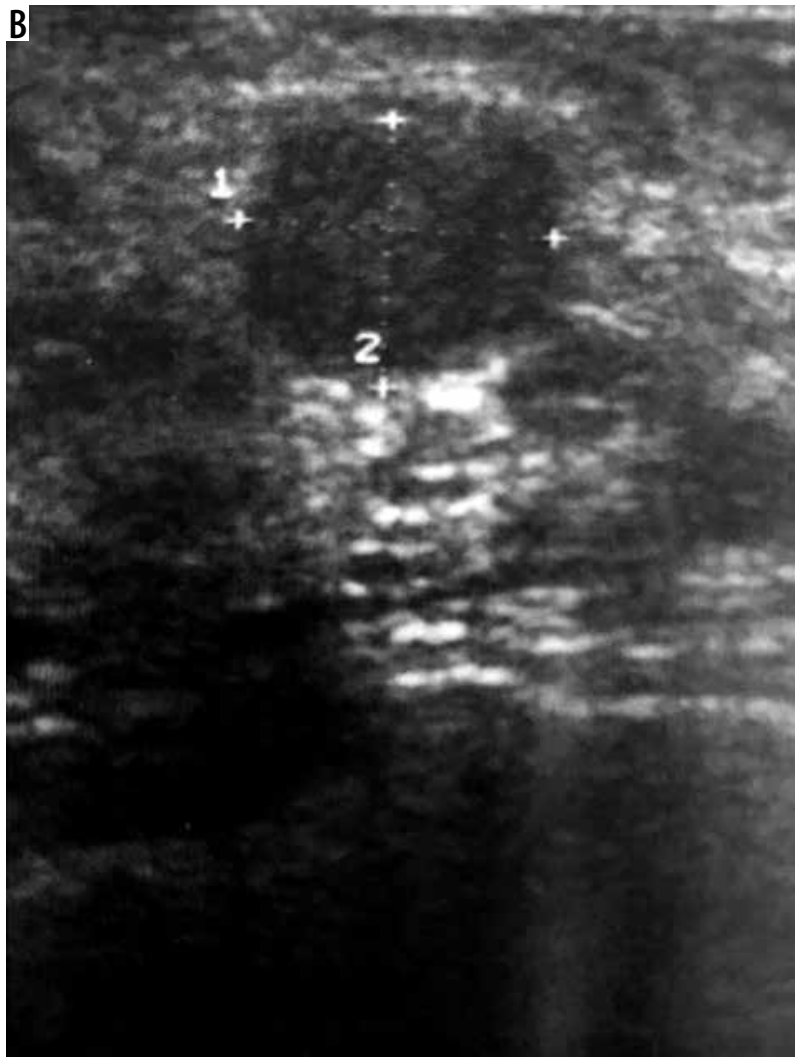

Figure 4. A) An asymptomatic woman and monographically dense breast. B) Stable cyst containing fine echoes (complex cyst) during follow-up ultrasounds of the same patient 

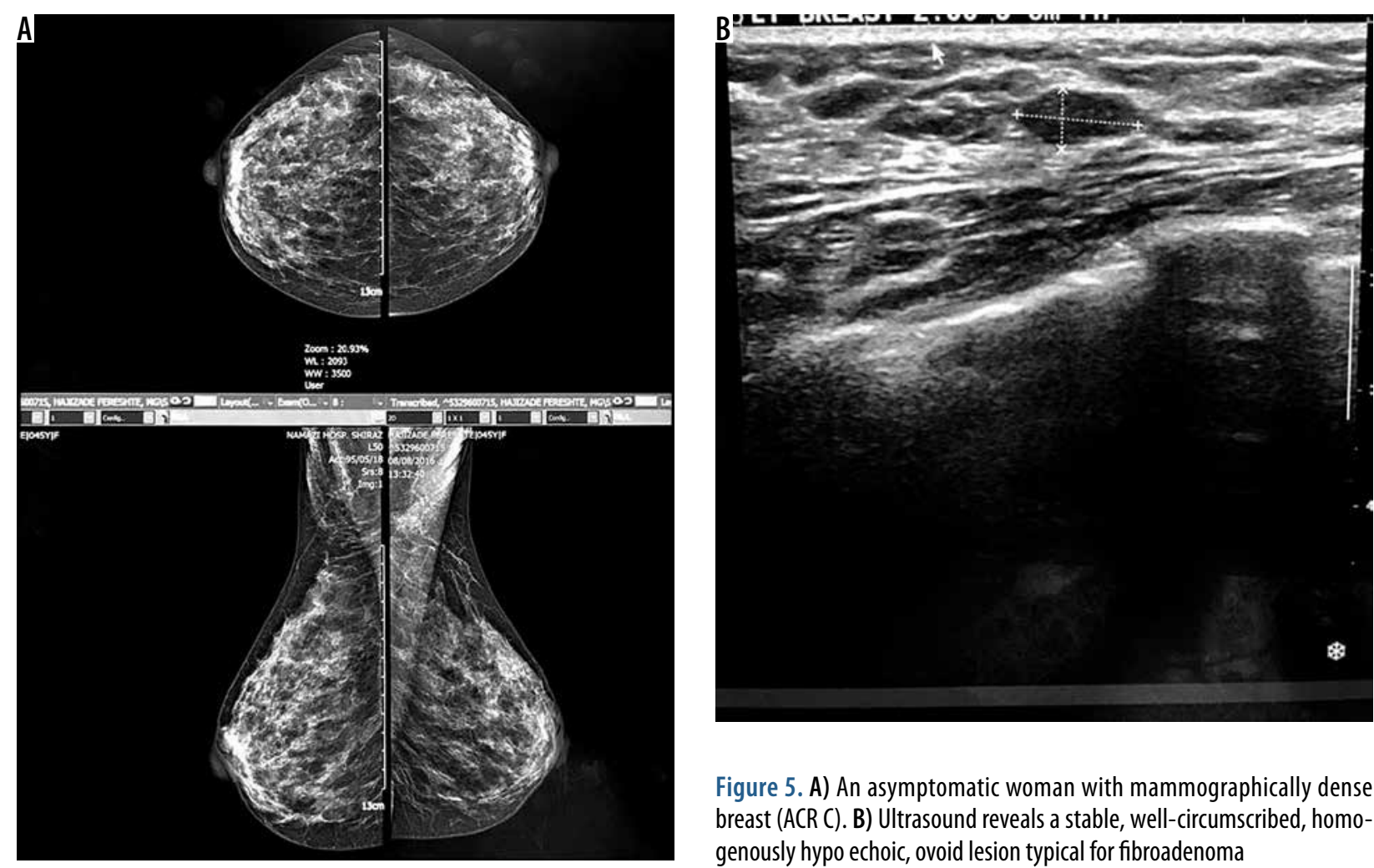

Figure 5. A) An asymptomatic woman with mammographically dense breast (ACR C). B) Ultrasound reveals a stable, well-circumscribed, homogenously hypo echoic, ovoid lesion typical for fibroadenoma
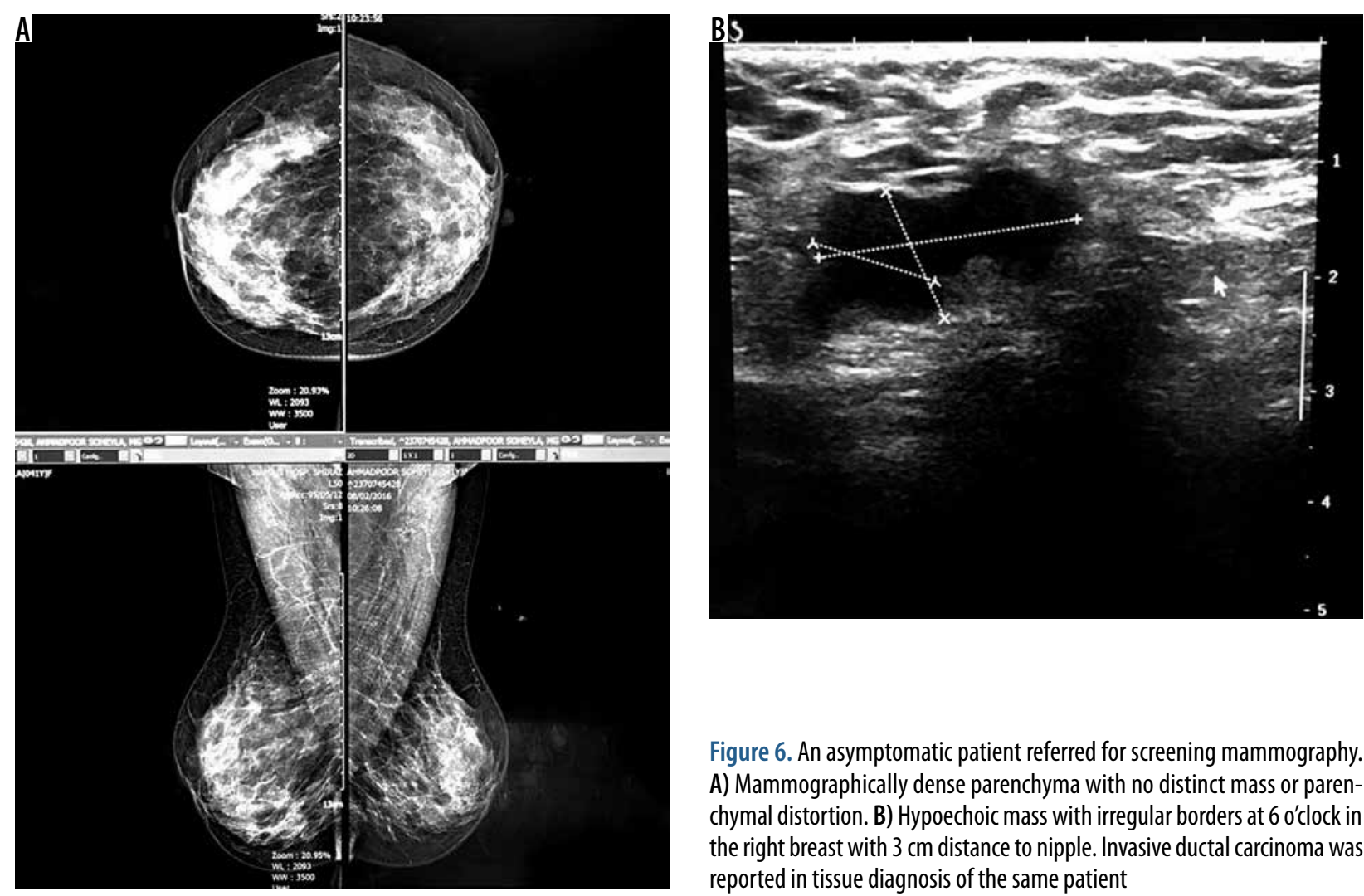

ovoid, without calcification or posterior shadowing and any changes during follow-up time) (Figure 5). Four cases underwent core needle biopsy sampling, of which three had "unshaped margin" or "indistinct border", the signs of

malignancy in sonography, and one had a large size despite its typical morphological sign of fibroadenoma. Biopsy result showed fibroadenoma in three of them and cancer in one case (Figure 6).

Figure 6. An asymptomatic patient referred for screening mammography. A) Mammographically dense parenchyma with no distinct mass or parenchymal distortion. B) Hypoechoic mass with irregular borders at $60^{\prime}$ clock in the right breast with $3 \mathrm{~cm}$ distance to nipple. Invasive ductal carcinoma was reported in tissue diagnosis of the same patient 
Table 2. Factors related to breast cancer

\begin{tabular}{|l|c|c|c|c|}
\hline Risk factors & Age (years) & Years of OCP usage & Frequency of breastfeeding & Frequency of pregnancy \\
\hline Minimum & 32 & 0 & 0 & 0 \\
\hline Maximum & 62 & 16 & 7 & 7 \\
\hline Mean \pm SD & $43.7 \pm 6.5$ & $1.6 \pm 3.1$ & $2.6 \pm 1.3$ & $2.7 \pm 1.4$ \\
\hline
\end{tabular}

Table 3. The relationship between risk factors and different mass lesions

\begin{tabular}{|l|c|c|c|c|c|c|c|}
\hline $\begin{array}{l}\text { Sono result } \\
\text { Risk factor }\end{array}$ & Normal & Benign lesion & Complicated cyst & Ductal ectasia & Fibroadenoma & Cancer & $p$-value \\
\hline Age (years) & $44.1 \pm 6.3$ & $43.3 \pm 6.1$ & $43.5 \pm 5.7$ & $45.2 \pm 7.8$ & $41.8 \pm 6.3$ & $37.0 \pm 0.0$ & 0.766 \\
\hline Pregnancy & $2.6 \pm 1.5$ & $2.9 \pm 1.3$ & $2.3 \pm 1.3$ & $3.0 \pm 0.8$ & $2.8 \pm 0.8$ & $3.0 \pm 0.0$ & 0.140 \\
\hline Breastfeed & $2.5 \pm 1.4$ & $2.8 \pm 1.3$ & $2.3 \pm 1.3$ & $3.0 \pm 0.8$ & $2.8 \pm 0.8$ & $3.0 \pm 0.0$ & 0.207 \\
\hline Oral contraceptive pill & $1.7 \pm 3.4$ & $1.4 \pm 2.8$ & $0.8 \pm 2.0$ & $1.0 \pm 2.0$ & $2.3 \pm 2.6$ & $4.0 \pm 0.0$ & 0.458 \\
\hline
\end{tabular}

Table 4. The relation between breast density and lesions

\begin{tabular}{|l|c|c|c|c|c|c|c|}
\hline $\begin{array}{l}\text { Sono result/ } \\
\text { Breast consistency }\end{array}$ & Normal & Benign lesion & Complicated cyst & Ductal ectasia & Fibroadenoma & Cancer & $p$-value \\
\hline Dense & $73.8 \%$ & $77.8 \%$ & $83.3 \%$ & $75.0 \%$ & $12.5 \%$ & $100.0 \%$ & 0.005 \\
\hline Very dense & $26.2 \%$ & $22.2 \%$ & $16.7 \%$ & $25.0 \%$ & $87.5 \%$ & $0.0 \%$ & \\
\hline
\end{tabular}

Table 2 shows the prevalence of different factors related to breast cancer.

The mean duration of OCP usage was $1.6 \pm 3.1$ years with a maximum duration of 16 years. The frequency of breastfeeding and pregnancy was $2.6 \pm 1.3$ and $2.7 \pm 1.4$ years, respectively.

Table 3 shows the relationship between different risk factors and sonography results in our subjects.

The mean age of the cases with ductal carcinoma was more than that of other lesions. Patients with complicated cysts had the minimum duration of pregnancy and breastfeeding, while this duration was greatest in ductal carcinoma. Patients with cancer and ductal ectasia had the longest duration of OCP usage. There was no significant relationship between different types of breast lesions and the prevalence of risk factors in our study.

Table 4 shows the relationship between the degree of breast density and different breast lesions that were detected in sonography; as the table shows, the prevalence of all lesions was higher in cases with dense breast compared to very dense breasts, except for the fibroadenoma, which was detected more in cases with very dense parenchyma. Our study shows that the prevalence of different breast lesions had a significant relationship with the density of the breast.

\section{Discussion}

The gold standard for breast cancer screening is mammography; however, sometimes there is a risk that mammography misses some cancers that are more common in dense breasts. With the advances in digital mammography and its increasing accuracy, the risk of error or failure to detect has decreased in women aged less than 50 years, dense breast women, and premenopausal or perimenopausal women [4]. In spite of all the benefits, digital mammography cannot overcome its fundamental restrictions; for example, poor view of non-calcified breast lesions and cancers as a result of overlying dense parenchyma $[4,11]$.

Breast ultrasonography as an adjuvant imaging modality for supplementary assessment of dense-breasted women might help to reveal mass lesions that might be missed by mammography [7].

Although mammographic screening has been successful in decreasing cancer mortality, Madjar et al. showed that mammography-based screening cannot resolve all breast problems. In dense-breasted women, the risk of breast cancer development is increased and the sensitivity of mammography is diminished [12].

In our study, $48.3 \%$ of the cases were diagnosed with at least one lesion in their sonography result; although $81 \%$ of them were benign lesions, other $19 \%$ needed follow-up or biopsy evaluation.

In the study by Min et al., US screening was offered to average-risk and dense-breasted women, resulting in higher detection rates of additional mammographically occult breast cancers [13].

Melnikow et al. suggested that additional screening of dense-breasted women would result in detection of more breast cancers (mostly invasive); however, it might be attributed to higher recurrence rates and additional biopsies [14].

In our study, nine cases were detected as complicated cyst and fibroadenoma and were followed up for 
36 months to monitor for any change. In all complicated cyst cases, the size of the lesions was reduced without developing any new lesion, and the fibroadenoma lesions showed no changes during follow-up.

In the study by Melnikow et al. complementary screening of women with dense breasts not only found more breast cancer but also increased the false-positive results. Hence, the effects of complimentary screening on breast cancer outcomes remains unclear [14].

In the study by Scheel et al. a technologist performed US breast screening for women with dense breasts, and after a year small occult breast cancers were detected with a detection rate of 3.2/1000 women screened [15]. In our study, the cancer detection rate was 1 in 207 women screened.

Okello recommends the routine performance of breast ultrasound scan for dense breasts categories 3 and 4 . Breast ultrasound scan leads to a considerable breast cancer detection rate among symptomatic dense-breasted women [16].

In the study by Kelikowske et al. the highest sensitivity of mammography was seen in high breast density women aged more than 50 years. The lowest sensitivity was observed in younger women, patients with a family history of breast cancer, or when the time between screenings was about two years. They also mentioned that, because not all dense-breasted women had high interval cancer rates in the study, the breast density should not be considered as the only criterion for deciding whether supplemental imaging is justified. In this study, there was a significant relationship between breast density and prevalence of different malignant and benign lesions [17].

In the study by Min et al. breast density was considered as an independent risk factor for breast cancer [13]. Devoli Disha et al. also considered breast density as a factor that increases the accuracy of sonography in the detection of mass lesions, compared to mammography [18].
Bakkun et al. mentioned the trigger of breast development is ovarian hormones and monthly menstruation regularly develops breast cell proliferation; considering this fact, OCP usage is considered as a risk factor that interferes with monthly menstrual cycles [19].

In different studies, age, OCP usage, breastfeeding duration, frequency of pregnancy, age of first pregnancy and breastfeeding, and family history and previous history of breast cancer were considered as factors that may affect the prevalence of different breast lesions. In our study, there was no significant relationship between these factors and the prevalence of different benign and malignant lesions.

\section{Conclusions}

A substantial number of occult mammographically breast lesions, either benign or malignant, could be detected by ultrasound in dense breast tissue.

However, larger volume is needed for study to evaluate the value and importance of additive ultrasound in mammographic dense parenchyma.

\section{Ethical statements}

All procedures followed were in accordance with the ethical standards of the responsible committee on human experimentation (institutional and national).

Informed consent was obtained from all patients for inclusion in the study.

\section{Conflict of interest}

The authors report no conflict of interest.

\section{References}

1. Key TJ, Verkasalo PK, Banks E. Epidemiology of breast cancer. Lancet Oncol 2001; 2: 133-140.

2. Zeinali Rafsanjani B, Mosleh-Shirazi MA, Faghihi R, et al. Breast cancer and its radiotherapeutic methods. Iranian J Med Phys 2012; 9: 75-85.

3. Chae EY, Kim HH, Cha JH, et al. Evaluation of screening whole-breast sonography as a supplemental tool in conjunction with mammography in women with dense breasts. J Ultrasound Med 2013; 32: 1573-1578.

4. Neal L, Tortorelli CL, Nassar A. Clinician's guide to imaging and pathologic findings in benign breast disease. Mayo Clin Proc 2010; 85: 274-279.

5. Chang JH, Vines E, Bertsch H, et al. The impact of a multidisciplinary breast cancer center on recommendations for patient management: the University of Pennsylvania experience. Cancer 2001; 91: 1231-1237.

6. Titus-Ernstoff L, Tosteson ANA, Kasales C, et al. Breast cancer risk factors in relation to breast density (United States). Cancer Causes Control 2006; 17: 1281-1290.
7. Salem DS, Kamal RM, Mansour SM, et al. Breast imaging in the young: the role of magnetic resonance imaging in breast cancer screening, diagnosis and follow-up. J Thorac Dis 2013; 5: S9-S18.

8. Melnikow J, Fenton JJ, Whitlock EP, et al. Supplemental screening for breast cancer in women with dense breasts: a systematic review for the U.S. Preventive Services Task Force. Ann Intern Med 2016; 164: 268-278.

9. Zarei F, Pishdad P, Hatami M, Zeinali-Rafsanjani B. Can breast ultrasound reduce patient's level of anxiety and pain? Ultrasound 2017; 25: 92-97.

10. Bae JM, Kim EH. Breast density and risk of breast cancer in Asian women: a meta-analysis of observational studies. J Prev Med Public Health 2016; 49: 367-375.

11. Ontario HQ. Ultrasound as an adjunct to mammography for breast cancer screening: a health technology assessment. Ontario Health Technology Assessment Series 2016; 16: 1. 
12. Madjar $\mathrm{H}$. Role of breast ultrasound for the detection and differentiation of breast lesions. Breast Care (Basel) 2010; 5: 109-114.

13. Chang JM, Koo HR, Moon WK. Radiologist-performed handheld ultrasound screening at average risk of breast cancer: results from a single health screening center. Acta Radiologica 2015; 56: 652-658.

14. Melnikow J, Fenton JJ, Whitlock EP, et al. U.S. Preventive Services Task Force Evidence Syntheses, formerly Systematic Evidence Reviews. Supplemental screening for breast cancer in women with dense breasts: a systematic review for the U.S. Preventive Service Task Force. Rockville (MD): Agency for Healthcare Research and Quality (US); 2016.

15. Scheel JR, Lee JM, Sprague BL, et al. Screening ultrasound as an adjunct to mammography in women with mammographically dense breasts. Am J Obstet Gynecol 2015; 212: 9-17.
16. Okello J, Kisembo H, Bugeza S, et al. Breast cancer detection using sonography in women with mammographically dense breasts. BMC Med Imaging 2014; 14: 41.

17. Kerlikowske K, Grady D, Barclay J, et al. Effect of age, breast density, and family history on the sensitivity of first screening mammography. JAMA 1996; 276: 33-38.

18. Devolli-Disha E, Manxhuka-Kerliu S, Ymeri H, et al. Comparative accuracy of mammography and ultrasound in women with breast symptoms according to age and breast density. Bosn J Basic Med Sci 2009; 9: 131-136.

19. Bakkum-Gamez JN, Laughlin SK, Jensen JR, et al. Challenges in the gynecologic care of premenopausal women with breast cancer. Mayo Clin Proc 2011; 86: 229-240. 